\title{
Characteristics and Outcomes of Elderly Patients Refused to ICU
}

\author{
María-Consuelo Pintado, ${ }^{1}$ Patricia Villa, ${ }^{1}$ Natalia González-García, ${ }^{2}$ \\ Jimena Luján, ${ }^{1}$ Rocío Molina, ${ }^{1}$ María Trascasa, ${ }^{1}$ Esther López-Ramos, \\ Cristina Martínez, ${ }^{1}$ José-Andrés Cambronero, ${ }^{1}$ and Raúl de Pablo ${ }^{1,3}$ \\ ${ }^{1}$ Intensive Care Unit, Hospital Universitario Príncipe de Asturias, Carretera Alcalá-Meco, s/n, Alcalá de Henares, \\ 28805 Madrid, Spain \\ ${ }^{2}$ Palliative Care Unit, Hospital Universitario Príncipe de Asturias, Alcalá de Henares, 28805 Madrid, Spain \\ ${ }^{3}$ Department of Medicine, University of Alcalá, Alcalá de Henares, 28871 Madrid, Spain
}

Correspondence should be addressed to María-Consuelo Pintado; consuelopintado@yahoo.es

Received 15 August 2013; Accepted 1 October 2013

Academic Editors: C. Czech, G. Onder, and W. Schupp

Copyright (C) 2013 María-Consuelo Pintado et al. This is an open access article distributed under the Creative Commons Attribution License, which permits unrestricted use, distribution, and reproduction in any medium, provided the original work is properly cited.

\begin{abstract}
Background. There are few data regarding the process of deciding which elderly patients are refused to ICU admission, their characteristics, and outcome. Methods. Prospective longitudinal observational cohort study. We included all consecutive patients older than 75 years, who were evaluated for admission to but were refused to treatment in ICU, during 18 months, with 12-month followup. We collected demographic data, ICU admission/refusal reasons, previous functional and cognitive status, comorbidity, severity of illness, and hospital and 12-month mortality. Results. 338 elderly patients were evaluated for ICU admission and 88 were refused to ICU (26\%). Patients refused because they were "too ill to benefit" had more comorbidity and worse functional and mental situation than those admitted to ICU; there were no differences in illness severity. Hospital mortality rate of the whole study cohort was $36.3 \%$, higher in patients "too ill to benefit" (55.6\% versus $35.8 \%, P<0.01$ ), which also have higher 1-year mortality ( $73.7 \%$ versus $42.5 \%, P<0.01)$. High comorbidity, low functional status, unavailable ICU beds, and age were associated with refusal decision on multivariate analysis. Conclusions. Prior functional status and comorbidity, not only the age or severity of illness, can help us more to make the right decision of admitting or refusing to ICU patients older than 75 years.
\end{abstract}

\section{Introduction}

There are no defined intensive care unit (ICU) admission criteria based on scientific evidence, so that decisions depend often on triaging doctor with marked variation in the rejection rates between different units [1-3]. With the increasing demand for critical care resources, often exceeding supply, its rationing is imposing [4]. This includes refusal of patients who are too ill or too well to benefit from critical care.

Several studies had shown that ICU refusal rates increase with increasing patient age, underlying disease, living in an institution, prior cognitive impairment, dependency, medical reason for admission, and bed availability [2-7].

Some authors have shown that outcomes of elderly patients after ICU admission are bad, with higher ICU mortality than younger patients, high 1-year mortality, and deterioration of functional autonomy and quality of life [3,
$8,9]$, supporting the idea of futility in the ICU admission of these patients. In contrast, other authors argue that patients should not be rejected solely because of age and consider that the most important determinant for ICU mortality is severity of illness, proposing that ICU practices should change [3-10].

The characteristics of elderly patients who were refused to ICU admission have been poorly analyzed. GarrousteOrgeas et al. [9] described that medical patient, full unit, age $>85$ years, and help needed for toileting were factors independently associated with refusal to ICU admission. However, in that study all refused patients were included in the logistic regression analysis, including patients too ill and too well to benefit from critical care.

Thus, we designed a prospective observational study to explore the outcome and characteristics of elderly patients refused to intensive care unit admission. The main objective 
of our study was to evaluate 1-year mortality of elderly patients refused to ICU. In addition, we also studied the characteristics of elderly patients referred to ICU admission, rates of refusal/admission to ICU and reasons motivating those decisions, factors associated to ICU refusal, hospital mortality, and number of rehospitalizations during the firstyear followup.

\section{Materials and Methods}

This is a prospective longitudinal observational cohort study conducted in a 14-bed Medical and Surgical ICU at the University Hospital Principe de Asturias in Alcalá de Henares, Spain.

All consecutive patients older than 75 years, who were evaluated for admission to but were refused to treatment in ICU, during 18 months from December 2009 to May 2011, were included.

Decisions regarding admission were made by ICU staff physician, who followed usual admission criteria, without predefined eligibility or exclusion criteria for those patients.

At the time of evaluation the following data were collected: age, sex, reason for triage, admission diagnosis, admission/refusal to ICU, referral site (emergency room, operating/recovery room, or ward), prior functional status (estimated by the Barthel Index (BI) [11]), prior cognitive status (determined according to the Cruz Roja Mental Scale (CRMS) [12]), comorbidity (measured by the Charlson Comorbidity Index (CCI) [13]), and evaluation of severity of illness at time of triage by the Acute Physiology, Age, and Chronic Health Evaluation Score (APACHE II) [14] and Sepsis-Related Organ Failure Assessment (SOFA) [15]. We defined functional independence as a punctuation above 60 on BI [11] and severe mental incapacity as a punctuation over 3 on Cruz Roja Mental Scale [16]. We defined 3 levels of comorbidity according to the CCI [13]: low (score: 0 or 1), medium (score: 2), and high (score: 3 or over). APACHE II score [14] was calculated for each patient using the data obtained at the time of evaluation. A one-year prospective followup of all patients included in the study was performed, including records of hospital stay, hospital mortality, vital status at 1 year, and number of rehospitalizations during that time. Outcome data were obtained by medical record (BI, CRMS, and CCI scores are shown in Supplementary Material available online at http://dx.doi.org/10.1155/2013/590837).

We also recorded reasons for refusal of ICU admission at triage using one-choice items, which included patients "too well to benefit" (inappropriate referral-these patients would not be considered for ICU admission and should never even have been referred), patients "too ill" (patients who were too ill on which ICU care was considered futile or who, it was felt, would derive some benefit from ICU care but insufficient benefit to be accorded a high enough priority to meet the admission threshold) and patient/family refused ICU admission; in this last option patients were excluded from study. When the reason for refusal of ICU given was "too ill," the triage physician recorded second reasons using multiple-choice items, which included patient "too old," high grade of comorbidity/severe underlying disease, severity of actual illness/futility and, prehospital disability.

The Institutional Ethics and Clinical Trials Committee approved the study protocol.

2.1. Statistical Analysis. Normal distribution of variables was assessed using the Kolmogorov-Smirnov test. Quantitative variables with normal distribution are expressed as mean \pm S.D.; nonnormal distribution variables are shown as medians and interquartile ranges. Qualitative variables are shown as percentages.

Comparisons between patients accepted and patients refused to ICU and comparisons between patients admitted to ICU and patients refused to ICU admission because they were "too ill to benefit," were based on the Student's $t$ test, Mann-Whitney test, and chi-square test, for quantitative variables with normal distribution, continuous variables with nonnormal distribution, and qualitative variables, respectively.

Level of statistical significance was set to $P$ values less than 0.05 and results are expressed with their $95 \%$ confidence intervals.

Logistic regression models were built for multivariate analysis to identify factors associated with ICU refusal. Univariate analysis of main variables registered at evaluation time (age, sex, reason for triage, referral site, beds availability, BI [11], CRMS [12], CCI [13], APACHE II [14], and SOFA [15]) was done. Predictor variables that were statistically significant when evaluated individually against ICU refusal were included in the forward stepwise multiple logistic regression analysis. The results were presented as odds ratios with the appropriate $95 \%$ confidence interval.

Statistical analysis was performed using SPSS 18.0 software (SPSS Inc., Chicago, IL).

\section{Results}

During the study period 338 patients older than 75 years were evaluated for ICU admission. Four patients refused ICU care and were excluded from the analysis.

The request came from the emergency room (56.3\%), medical or surgical wards $(33.5 \%)$, and operating/recovery room $(10.2 \%)$.

The baseline characteristics of all patients evaluated for ICU admission are shown in Table 1 and the main reason for ICU evaluation is shown in Table 2. There were statistical significant differences between the group of accepted versus rejected patients in respiratory, infectious, and renal/genitourinary reasons for the ICU triage decision. The percentage of patients triaged due to respiratory support was higher in the refused group of patients, whereas there was higher rate of patients with infectious reason for critical care on patients admitted to ICU.

Of 334 patients who underwent screening for eligibility, 177 patients were initially admitted to ICU (18 patients were transferred to another ICU in another hospital due to lack of available beds in our unit) and 18 patients refused for admission due to being "too well to benefit" were admitted 
TABLE 1: Clinical characteristics of the patients at first ICU.

\begin{tabular}{lc}
\hline Patients characteristics & $\begin{array}{c}\text { All patients } \\
\text { evaluated for ICU } \\
\text { admission }(n=334)\end{array}$ \\
\hline Age $^{*}$ & $81(78-85)$ \\
81-85 years & $143(42.8 \%)$ \\
& $126(37.7 \%)$ \\
Gender: male & $65(19.5 \%)$ \\
Charlson Comorbidity Index & $168(50.3 \%)$ \\
Comorbidity & $2(1-3)$ \\
Low & \\
Medium & $141(42.5 \%)$ \\
High & $90(27.1 \%)$ \\
Barthel Index* & $101(30.4 \%)$ \\
Completely independent & $95(65-100)$ \\
Mild dependence & $156(49.7 \%)$ \\
Moderate dependence & $95(30.3 \%)$ \\
Severe dependence & $34(10.8 \%)$ \\
Completely dependent & $10(3.2 \%)$ \\
Functional dependence (Barthel Index $<60)$ & $19(6.1 \%)$ \\
Cruz Roja Mental Scale & $63(20.1 \%)$ \\
Completely normal & \\
Light disorders of disorientation in time & $66(21 \%)$ \\
Disorientation in time & $35(11.1 \%)$ \\
Complete disorientation & $2(6 \%)$ \\
Complete mental disorder & $3(1 \%)$ \\
Dementia very evident & $4(1.3 \%)$ \\
APACHE II* & $7(2.09 \%)$ \\
\hline SOFA & $15(11-22)$ \\
Deve mental incapacity $(C R M S ~$ & $4(1-7)$ \\
\hline
\end{tabular}

Data are shown as number (percent), except for those marked with * that are shown as median $\left(\mathrm{P}_{25}-\mathrm{P}_{75}\right)$.

to the ICU within 24 hours after a second evaluation. Thus, 195 patients were included in the study. Among the 139 patients refused to ICU admission, 51 patients (36.7\%) were refused because they were "too well to benefit" and 88 patients (63.3\%) because they were "too ill" (Figure 1). Causes for refusal of patients considered "too ill" were being "too old" in $54.5 \%$ of patients, high grade of comorbidity/severe underlying disease in $68.2 \%$, severity of actual illness/futility in $51.1 \%$, and prehospital disability in $37.5 \%$. Two or more causes were present in $71.5 \%$ of patients.

Outcomes of patients who were candidates to ICU are shown in Table 3. Hospital mortality rate of the whole study cohort was $42.1 \%$ : $35.8 \%$ in the admitted group, $55.6 \%$ in the "too ill to benefit" group, and 5.9\% in the 51 patients considered "too well to benefit."

There was higher 1-year mortality among patients refused to ICU $(71.3 \%$ versus $41.0 \%$ in patients admitted to ICU, $P<0.01$ ) (Table 3). This 1-year mortality was higher than

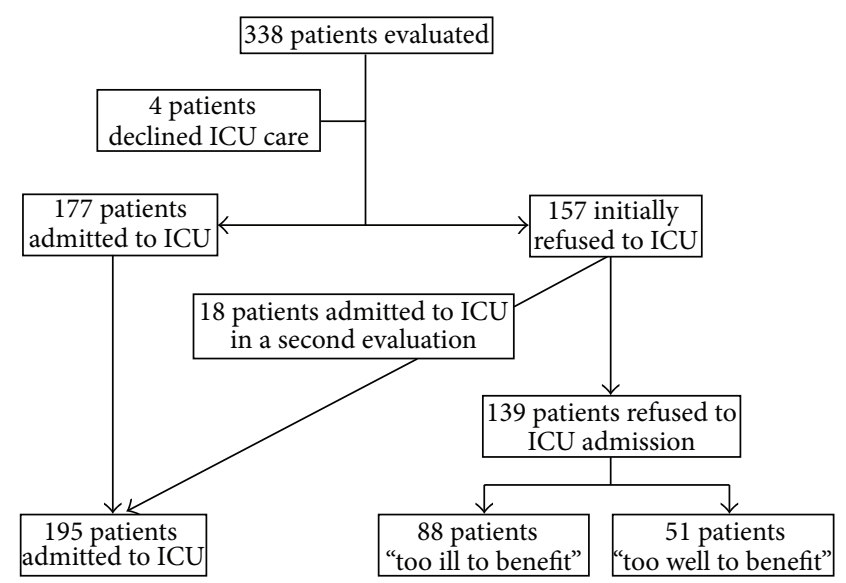

FIGURE 1: Flow chart of inclusion and exclusion patients.

expected according to their CCI (median CCI [13] of 3 correlates with an expected 1-year mortality of 52\%) [13].

We next investigated the differences between the baseline characteristics of patients admitted to ICU and of patients refused to ICU admission because they were "too ill to benefit." Patients refused to ICU admission and "too ill to benefit" were older (mean age of $82(79-85)$ years versus 81 (78-84) years in admitted to ICU patients, $P=0.012)$ and had more comorbidity (mean CCI [13] $3(2-4)$ versus $1(0-$ 2 ) in patients admitted to ICU, $P<0.001$ ), worse functional situation (mean BI [11] 67.50 (40-100) versus 100 (85-100) of patients admitted to ICU, $P<0.001)$, and worse prior mental status (none of patients admitted to ICU had a severe mental incapacity versus $5.6 \%$ in rejected patients, $P=0.004$ ). Conversely, there was no difference in gender or severity of illness measured by SOFA [15] and APACHE II [14] scores between patients refused or admitted to ICU (Table 4).

To assess the impact of clinical variables associated with the decision to refuse ICU admission in "too ill to benefit" patients, we performed a logistic regression analysis. We identify that comorbidity according to the CCI [13], prior functional status measured by the Barthel Index [11], age, and no available ICU beds are factors independently associated with refusal of ICU admission (Table 5).

\section{Discussion}

In this prospective single-center study, we have found that, among patients older than 75 years, those refused to ICU admission have higher hospital mortality and 1-year mortality than patients admitted to ICU. Patient-related factors associated with refusal to ICU admission were more related to age, or prior functional or comorbidity status, than to the severity of illness at time of triage.

We observed that the rate of refusal of elderly patients (older than 75 years) to our ICU (41.62\%) was slightly higher than the rate described in a previous study in patients older than 74 years (25\%) [3]. However, this rate was lower than those found in two French studies with octogenarians patients, where the rate was $73 \%$ [9] and $88 \%$ [1], although a 
TABLE 2: Main reason for patient evaluation at first ICU triage.

\begin{tabular}{lccc}
\hline & $\begin{array}{c}\text { Accepted to ICU } \\
n=195(57.7 \%)\end{array}$ & $\begin{array}{c}\text { Refused to ICU } \\
n=139(42.3 \%)\end{array}$ & $P$ value accepted versus refused \\
\hline Cardiac arrest & $10(5.1 \%)$ & $10(7.2 \%)$ & 0.433 \\
Cardiovascular & $112(57.4 \%)$ & $72(51.8 \%)$ & 0.307 \\
Respiratory & $8(4.1 \%)$ & $22(15.8 \%)$ & 0.000 \\
Neurologic & $6(3.1 \%)$ & $9(6.5 \%)$ & 0.139 \\
Intoxications & $3(1.5 \%)$ & $4(2.9 \%)$ & 0.400 \\
Digestive & $4(2.1 \%)$ & $2(1.4 \%)$ & 0.678 \\
Surgical & $7(3.6 \%)$ & $2(1.4 \%)$ & 0.231 \\
Infectious & $43(22.1 \%)$ & $14(10.1 \%)$ & 0.004 \\
Renal/genitourinary & $0(0 \%)$ & $4(2.9 \%)$ & 0.017 \\
Others & $2(1 \%)$ & $0(0 \%)$ & 0.513 \\
\hline
\end{tabular}

Data are shown as number (percentage).

TABLE 3: Outcomes of patients who were candidates to ICU.

\begin{tabular}{|c|c|c|c|}
\hline & $\begin{array}{l}\text { Patients admitted } \\
\text { to ICU }(n=195)\end{array}$ & $\begin{array}{l}\text { Patients refused } \\
\text { to ICU }(n=88)\end{array}$ & $P$ value \\
\hline Hospital mortality & $\begin{array}{l}70 / 195 \\
35.8 \%\end{array}$ & $\begin{array}{l}49 / 88 \\
55.6 \%\end{array}$ & 0.002 \\
\hline Length of hospital stay ${ }^{*}$ & $11(6-21.5)$ & $7.5(2-12.25)$ & 0.000 \\
\hline Mortality during 1-year followup & $\begin{array}{l}10 / 125 \\
8.0 \%\end{array}$ & $\begin{array}{l}13 / 38 \\
34.2 \%\end{array}$ & 0.000 \\
\hline 1-year mortality & $\begin{array}{l}80 / 195 \\
41.0 \%\end{array}$ & $\begin{array}{l}62 / 87 \\
71.3 \%\end{array}$ & 0.000 \\
\hline$\%$ patients who were rehospitalized during 1-year followup & $\begin{array}{l}68 / 115 \\
59.1 \%\end{array}$ & $\begin{array}{l}17 / 38 \\
44.7 \%\end{array}$ & 0.122 \\
\hline
\end{tabular}

Data are shown as number (percent), except for those marked with * that are shown as median $\left(\mathrm{P}_{25}-\mathrm{P}_{75}\right)$.

great variability between centers was observed. This finding may be influenced by the fact that 51 of 139 patients $(36.69 \%)$ were considered "too well to benefit" or by the variation in hospital organization across countries.

Analysis of the hospital mortality of patients refused to ICU because they were "too well" or "too ill" to benefit revealed that hospital mortality of those patients was $5.9 \%$ and $56.3 \%$, respectively. As expected, patients who were refused to admission due to futility had higher observed mortality than those admitted. In both groups of refused patients, we found lower hospital mortality than that described in previous studies. Garrouste-Orgeas et al. [9] found higher hospital mortality rates than in our study (17.6\% in patients "too well" and $70.8 \%$ in those "too ill"), although their study only included octogenarians. Similar rates were reported in the study of Sprung et al. [3] in patients of 75 years old and older (20\% in patients "too well" and $77.2 \%$ in those "too ill"), whereas Joynt et al. [17] described a hospital mortality of $90 \%$ in patients refused to ICU admission due to futility and of $8 \%$ in patients considered too well to benefit from ICU treatment.

We have an unexpected high rate of survival of patients refused to ICU due to being too ill (44\%). In contrast, Joynt et al. described a low rate of hospital survival of $10 \%$ in patients refused to ICU because of perceived futility [17]. This is in agreement with the low relevance in our case of the severity of illness as variable for the refusal decision. These data highlight the difficulty of assessing futility for intensivists. The 1-year mortality rate in our study was $41 \%$ in admitted patients, which is lower than that described before in elderly patients $(42-70 \%)[8,9]$. Patients refused to ICU due to being "too ill to benefit" had not only a higher hospital mortality, but also a higher mortality rate during 1-year followup as in previous studies $[8,9]$.

Trying to understand the reasons to adopt decisions of refusing ICU admission, we investigated the factors associated with futility. Factors associated with patients "too well to benefit" were not analyzed, because these patients were really inappropriate referrals. As in previous studies, we found that patients refused to ICU have a worse previous functional (BI [11]) and cognitive (Cruz Roja Mental Scale [12]) status, higher comorbidity (CCI [13]), but not higher severity of illness at time of triage (APACHE II [14] and SOFA [15] scores), compared with those accepted to ICU $[1,3,7,9]$. We found that among all the patients referred for ICU admission, $20.1 \%$ of patients were dependent (defined as having a BI [11] lower than 60), but only $2.09 \%$ had a severe mental incapacity. There are few data about this point. GarrousteOrgeas et al. [18] report that only $6.9 \%$ of adult patients referred to ICU admission were dependent at the time of triage. Daubin et al. [19] in their study about predictors of 
TABLE 4: Baseline characteristics of patients who were candidates to ICU.

\begin{tabular}{|c|c|c|c|}
\hline & $\begin{array}{c}\text { Patients admitted to UCI } \\
n=195(68.9 \%)\end{array}$ & $\begin{array}{c}\text { Patients refused to ICU } \\
n=88(31.0 \%)\end{array}$ & $P$ value \\
\hline Age $^{*}$ & $81(78-84)$ & $82(79-85)$ & 0.012 \\
\hline$>75-80$ years & $93(47.7 \%)$ & $29(33 \%)$ & \\
\hline $81-85$ years & $66(33.8 \%)$ & $38(43.2 \%)$ & \\
\hline$>85$ & $36(18.5 \%)$ & $21(23.9 \%)$ & \\
\hline Gender: male & $97(49.7 \%)$ & $47(53.4 \%)$ & 0.568 \\
\hline Charlson Comorbidity Index* & $1(0-2)$ & $3(2-4)$ & 0.000 \\
\hline Comorbidity & & & 0.000 \\
\hline Low & $101(52.1 \%)$ & $18(20.5 \%)$ & \\
\hline Medium & $53(27.3 \%)$ & $24(27.3 \%)$ & \\
\hline High & $40(20.6 \%)$ & $46(52.3 \%)$ & \\
\hline Barthel Index* & $100.0(85-100)$ & $67.5(40-100)$ & 0.000 \\
\hline Completely independent & $95(48.7 \%)$ & $27(30.7 \%)$ & \\
\hline Mild dependence & $65(33.3 \%)$ & $23(26.1 \%)$ & \\
\hline Moderate dependence & $11(5.6 \%)$ & $18(20.5 \%)$ & \\
\hline Severe dependence & $2(1 \%)$ & $6(6.8 \%)$ & \\
\hline Completely dependent & $22(11.3 \%)$ & $14(15.9 \%)$ & \\
\hline Functional dependence (Barthel Index < 60) & $35(17.9 \%)$ & $38(43.2 \%)$ & 0.000 \\
\hline Cruz Roja Mental Scale & & & 0.001 \\
\hline Completely normal & $132(74.6 \%)$ & $40(46.0 \%)$ & \\
\hline Light disorders of disorientation in time & $29(16.4 \%)$ & $28(32.2 \%)$ & \\
\hline Disorientation in time & $16(9.0 \%)$ & $13(14.9 \%)$ & \\
\hline Complete disorientation & $0(0 \%)$ & $2(2.3 \%)$ & \\
\hline Complete mental disorder & $0(0 \%)$ & $3(3.4 \%)$ & \\
\hline Dementia very evident & $0(0 \%)$ & $1(1.1 \%)$ & \\
\hline Severe mental incapacity (CRMS > 3) & $0(0 \%)$ & $4(5.6 \%)$ & 0.004 \\
\hline APACHE II & $17(11-23)$ & $17(11-24)$ & 0.660 \\
\hline SOFA $^{*}$ & $5(1-8.25)$ & $4(2-6)$ & 0.301 \\
\hline
\end{tabular}

Data are shown as number (percentage), except for those marked with ${ }^{*}$ that are shown as median $\left(\mathrm{P}_{25}-\mathrm{P}_{75}\right)$.

mortality and short-term physical and cognitive dependence in critically ill persons of 75 years and older admitted in ICU report that only $57 \%$ and $40 \%$ of patients were completely physically dependent measured by the Katz Index of Activity of Daily Living [20] and cognitive independent measured by individual components of the Lawton Index of Daily Living [21], respectively, and $1 \%$ and $7 \%$ were completely dependent. In our study, the $48.7 \%$ in the admitted group and $30.7 \%$ in the refused group were completely physically independent (defined as BI [11] 100); $11.3 \%$ and $15.9 \%$, respectively, were completely physically dependent (defined as BI [11] lower than 20). Completely normal cognitive situation was found in $74.6 \%$ in the admitted group and in $46.0 \%$ in the refused group, whereas severe mental incapacity was found in $0 \%$ and $5.6 \%$ in the admitted and refused groups respectively.

We found that age, comorbidity, prior functional status, and full unit were independent variables associated with ICU refusal. There are few data about these points. GarrousteOrgeas et al. found that age, medical status, and full unit were the factors associated with refusal in octagenarians patients
[9]. Iapichino et al. found that age and more than one ICU triage were associated with ICU refusal in general population [7]. Joynt et al. found that age, diagnostic category, severity of illness, and operative factors were important factors in ICU refusal [17]. We thought that we did not have triage (the process of sorting referred patients in order of priority $[22,23])$ as a reason for the decision, because if we thought that the patient needed admission to the ICU, the patient was transferred to another hospital. However, we found a strong effect of bed availability for refusing patients to ICU admission. This finding is almost always found by other authors $[5,18,24,25]$.

This study had several limitations. First, it was conducted in a single centre and our results may not be applicable to other hospitals or healthcare systems. Wide variability from one hospital to another has been described [1]. Second, it was observational and the lack of randomization is a source of inaccuracy. Third, we did not measure quality of life in survivors at one-year followup and self-dependency and quality of life may be poorer than in general population [9] 
TABLE 5: Factors associated with ICU refusal.

\begin{tabular}{|c|c|c|}
\hline \multicolumn{3}{|c|}{ Univariate analysis } \\
\hline & Odds ratio $(95 \% \mathrm{CI})$ & $P$ value \\
\hline Age & $1.07(1.01-1.14)$ & 0.011 \\
\hline Sex (male) & $0.83(0.52-1.43)$ & 0.568 \\
\hline APACHE II & $1.01(0.98-1.04)$ & 0.414 \\
\hline SOFA & $0.93(0.87-1.00)$ & 0.056 \\
\hline Charlson Comorbidity Score & $1.58(1.33-1.89)$ & 0.000 \\
\hline Barthel Index & $0.97(0.96-0.98)$ & 0.000 \\
\hline Cruz Roja Mental Scale & $2.12(1.52-2.96)$ & 0.000 \\
\hline No beds available & $0.37(1.13-0.99)$ & 0.040 \\
\hline \multicolumn{3}{|l|}{ Referral site } \\
\hline \multicolumn{3}{|l|}{ Reason for patient evaluation } \\
\hline Cardiac arrest & $2.37(0.94-5.92)$ & 0.061 \\
\hline Cardiovascular & $0.48(2.29-0.81)$ & 0.001 \\
\hline Respiratory & $7.32(3.09-1.32)$ & 0.000 \\
\hline Neurologic & $2.72(0.88-8.35)$ & 0.079 \\
\hline Intoxications & $3.05(0.67-13.91)$ & 0.150 \\
\hline Digestive & $0.55(0.06-4.98)$ & 0.591 \\
\hline Surgical & $0.31(0.04-2.55)$ & 0.270 \\
\hline Infectious & $0.40(0.18-0.87)$ & 0.020 \\
\hline \multicolumn{3}{|c|}{ Multivariate analysis } \\
\hline & Odds ratio $(95 \% \mathrm{CI})$ & $P$ value \\
\hline Age & $1.09(1.01-1.17)$ & 0.018 \\
\hline Charlson Comorbidity Index & $1.58(1.31-1.91)$ & 0.000 \\
\hline Barthel Index & $0.97(0.96-0.98)$ & 0.000 \\
\hline No beds available & $0.26(0.08-0.81)$ & 0.021 \\
\hline
\end{tabular}

Our strengths are that it is a study with a prospective observational design, with a large enrolment period, realized exclusively in elderly patients (older than 75 years).

\section{Conclusions}

In conclusion, not only age, but also prior functional status measured by the Barthel Index [11] and the degree of comorbidity measured by the Charlson Comorbidity Index [13] can help us to make the right decision of admitting or refusing to ICU patients older than 75 years old. However, we need more multicenter and randomized studies to improve our ICU admission criteria.

\section{Conflict of Interests}

None of the authors received financial support for participating in this study.

\section{Acknowledgment}

The authors want to thank all staff and patients of Principe de Asturias University Hospital that have worked or collaborated selflessly in this study. This paper has not been presented previously in poster or other sessions.

\section{References}

[1] A. Boumendil, D. C. Angus, A.-L. Guitonneau et al., "Variability of intensive care admission decisions for the very elderly," PLoS ONE, vol. 7, no. 4, Article ID e34387, 2012.

[2] D. L. Edbrooke, C. Minelli, G. H. Mills et al., "Implications of ICU triage decisions on patient mortality: a cost-effectiveness analysis," Critical Care, vol. 15, no. 1, article R56, 2011.

[3] C. L. Sprung, A. Artigas, J. Kesecioglu et al., "The Eldicus prospective, observational study of triage decision making in European intensive care units. Part II: intensive care benefit for the elderly," Critical Care Medicine, vol. 40, no. 1, pp. 132-138, 2012.

[4] N. S. Ward and M. M. Levy, "Rationing and critical care medicine," Critical Care Medicine, vol. 35, no. 2, pp. S102-S105, 2007.

[5] M. Garrouste-Orgeas, L. Montuclard, J.-F. Timsit et al., "Predictors of intensive care unit refusal in French intensive care units: a multiple-center study," Critical Care Medicine, vol. 33, no. 4, pp. 750-755, 2005.

[6] J. Reignier, R. Dumont, S. Katsahian et al., "Patient-related factors and circumstances surrounding decisions to forego lifesustaining treatment, including intensive care unit admission refusal," Critical Care Medicine, vol. 36, no. 7, pp. 2076-2083, 2008.

[7] G. Iapichino, D. Corbella, C. Minelli et al., "Reasons for refusal of admission to intensive care and impact on mortality," Intensive Care Medicine, vol. 36, no. 10, pp. 1772-1779, 2010.

[8] E. Sacanella, J. M. Pérez-Castejón, J. M. Nicolás et al., "Functional status and quality of life 12 months after discharge from a medical ICU in healthy elderly patients: a prospective observational study," Critical Care, vol. 15, no. 2, article R105, 2011.

[9] M. Garrouste-Orgeas, J.-F. Timsit, L. Montuclard et al., "Decision-making process, outcome, and 1-year quality of life of octogenarians referred for intensive care unit admission," Intensive Care Medicine, vol. 32, no. 7, pp. 1045-1051, 2006.

[10] A. Boumendil, D. Somme, M. Garrouste-Orgeas, and B. Guidet, "Should elderly patients be admitted to the intensive care unit?" Intensive Care Medicine, vol. 33, no. 7, pp. 1252-1262, 2007.

[11] F. I. Mahoney and D. W. Barthel, "Functional evaluation: the Barthel Index," Maryland State Medical Journal, vol. 14, pp. 6165, 1965.

[12] F. G. Llera and A. García Antón, "Ayuda a domicilio. Aspectos médicos en Geriatría," Revista Española de Geriatría y Gerontología, vol. 7, pp. 339-346, 1972.

[13] M. E. Charlson, P. Pompei, K. A. Ales, and C. R. MacKenzie, "A new method of classifying prognostic comorbidity in longitudinal studies: development and validation," Journal of Chronic Diseases, vol. 40, no. 5, pp. 373-383, 1987.

[14] W. A. Knaus, E. A. Draper, D. P. Wagner, and J. E. Zimmerman, "APACHE II: a severity of disease classification system," Critical Care Medicine, vol. 13, no. 10, pp. 818-829, 1985.

[15] J.-L. Vincent, A. de Mendonça, F. Cantraine et al., "Use of the SOFA score to assess the incidence of organ dysfunction/failure in intensive care units: results of a multicenter, prospective study," Critical Care Medicine, vol. 26, no. 11, pp. 1793-1800, 1998.

[16] M. J. Cabañero-Martínez, J. Cabrero-García, M. RichartMartínez, and C. L. Muñoz-Mendoza, "Revisión estructurada de las medidas de actividades de la vida diaria en personas mayores," Revista Española de Geriatría y Gerontología, vol. 43, no. 5, pp. 271-283, 2008. 
[17] G. Joynt, C. Gomersall, P. Tan, A. Lee, C. Cheng, and E. Wong, "Prospective evaluation of patients refused admission to an intensive care unit: triage, futility and outcome," Intensive Care Medicine, vol. 27, no. 9, pp. 1459-1465, 2001.

[18] M. Garrouste-Orgeas, L. Montuclard, J.-F. Timsit, B. Misset, M. Christias, and J. Carlet, "Triaging patients to the ICU: a pilot study of factors influencing admission decisions and patient outcomes," Intensive Care Medicine, vol. 29, no. 5, pp. 774-781, 2003.

[19] C. Daubin, S. Chevalier, A. Séguin et al., "Predictors of mortality and short-term physical and cognitive dependence in critically ill persons 75 years and older: a prospective cohort study," Health and Quality of Life Outcomes, vol. 9, article 35, 2011.

[20] S. Katz, T. D. Downs, H. R. Cash, and R. C. Grotz, "Progress in development of the index of ADL," Gerontologist, vol. 10, no. 1, pp. 20-30, 1970.

[21] P. Barberger-Gateau, D. Commenges, M. Gagnon, L. Letenneur, C. Sauvel, and J.-F. Dartigues, "Instrumental activities of daily living as a screening tool for cognitive impairment and dementia in elderly community dwellers," Journal of the American Geriatrics Society, vol. 40, no. 11, pp. 1129-1134, 1992.

[22] C. L. Sprung, M. Danis, M. A. Bailey et al., "Attitudes of critical care medicine professionals concerning distribution of intensive care resources," Critical Care Medicine, vol. 22, no. 2, pp. 358-362, 1994.

[23] C. Kohlenberger, C. L. Sprung, M. Danis et al., "Consensus statement on the triage of critically ill patients," Journal of the American Medical Association, vol. 271, no. 15, pp. 1200-1203, 1994.

[24] E. Azoulay, F. Pochard, S. Chevret et al., "Compliance with triage to intensive care recommendations," Critical Care Medicine, vol. 29, no. 11, pp. 2132-2136, 2001.

[25] C. L. Sprung, D. Geber, L. A. Eidelman et al., "Evaluation of triage decisions for intensive care admission," Critical Care Medicine, vol. 27, no. 6, pp. 1073-1079, 1999. 


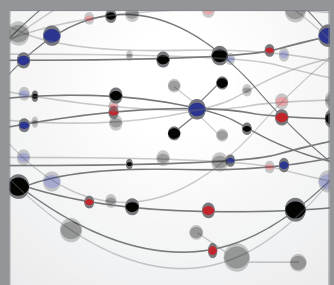

The Scientific World Journal
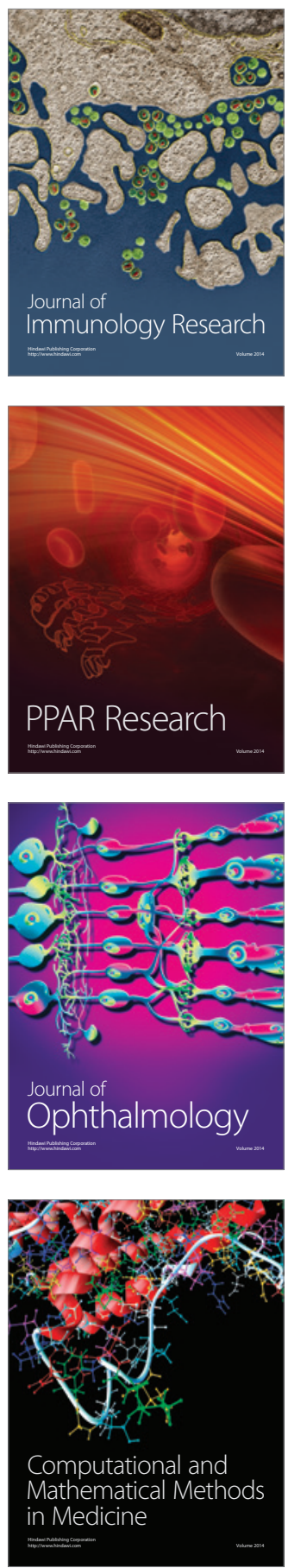

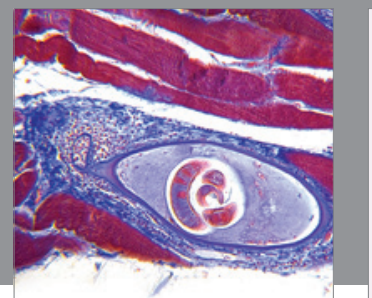

Gastroenterology

Research and Practice
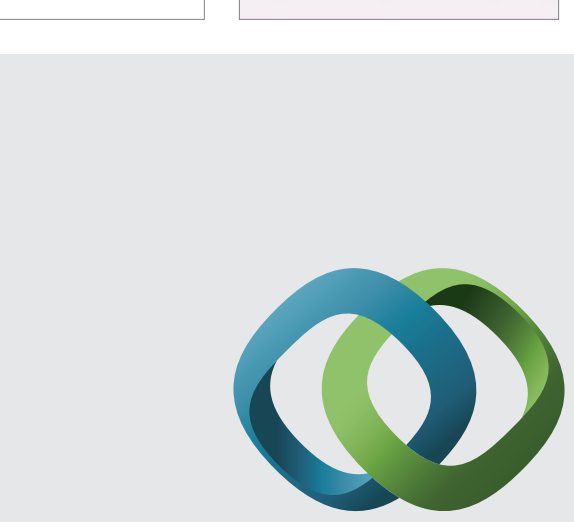

\section{Hindawi}

Submit your manuscripts at

http://www.hindawi.com
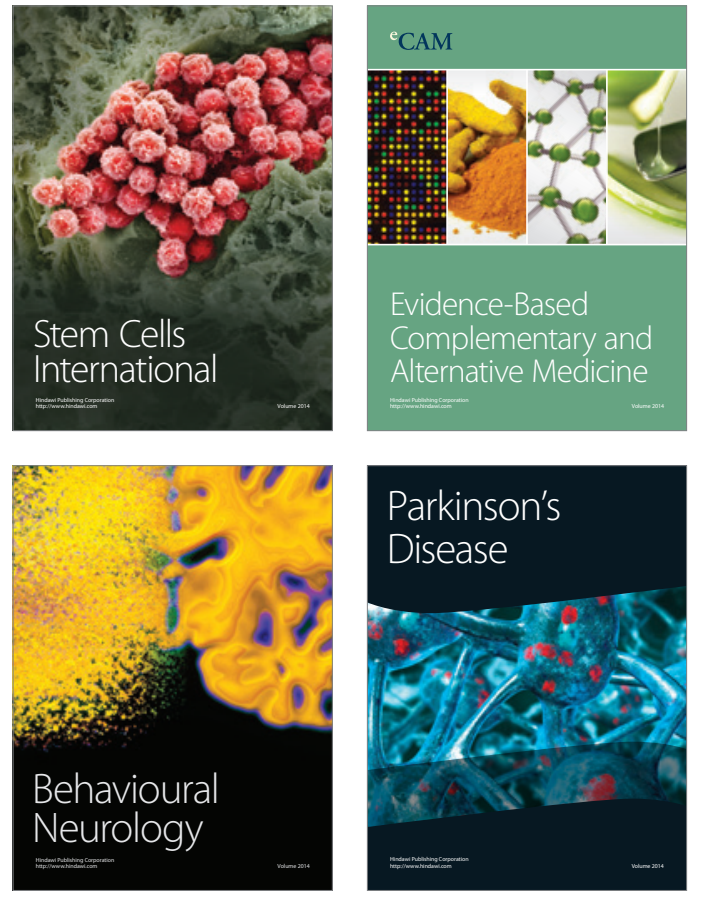
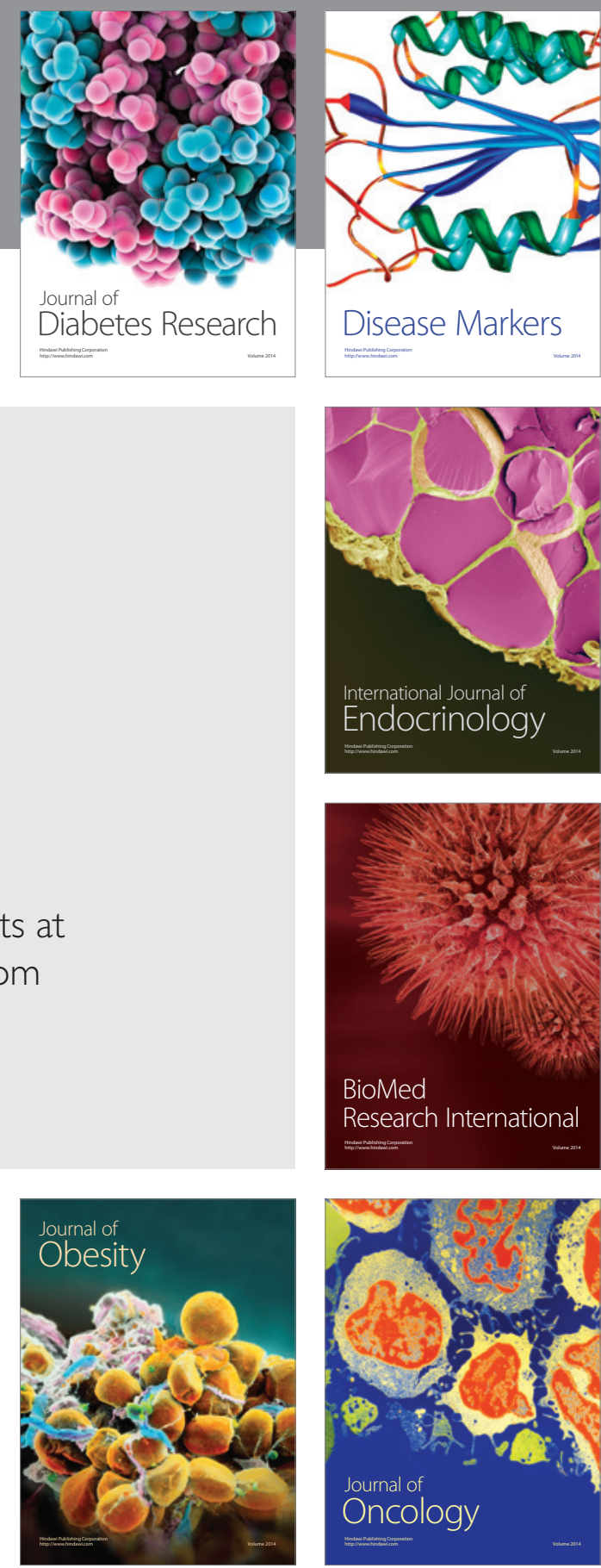

Disease Markers
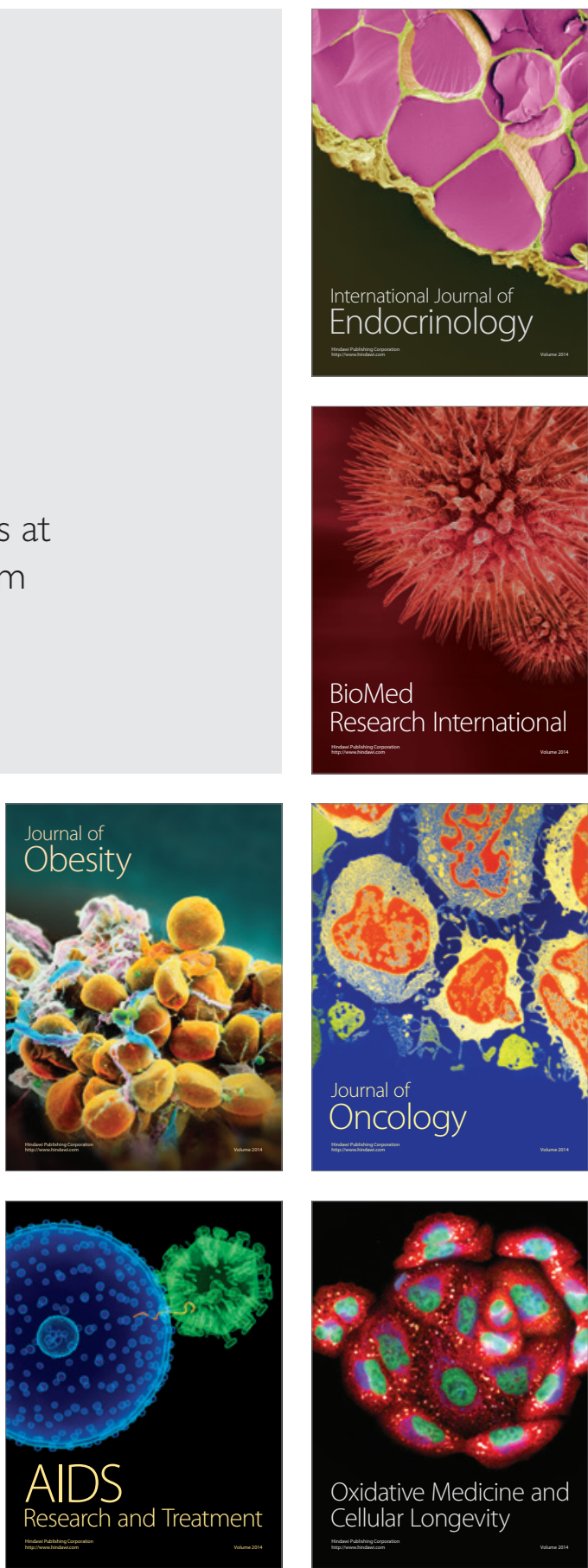